Prism Signal Processing for Machine Condition Monitoring I: Design and Simulation

Manus Henry, Vladimir Sinitsin

Accepted for 1st IEEE International Conference on Industrial Cyber-Physical Systems (ICPS 2018), Saint Petersburg, Russia, May 15-18, 2018.

(C) 2018 IEEE. Personal use of this material is permitted. Permission from IEEE must be obtained for all other uses, including reprinting/republishing this material for advertising or promotional purposes, collecting new collected works for resale or redistribution to servers or lists, or reuse of any copyrighted component of this work in other works. 


\section{Prism Signal Processing for Machine Condition Monitoring I: Design and Simulation}

\author{
Manus Henry ${ }^{\mathrm{a}, \mathrm{b}}$ \\ ${ }^{a}$ Department of Engineering Science \\ University of Oxford \\ Oxford, OX1 3PJ, UK. \\ manus.henry@eng.ox.ac.uk
}

\author{
Vladimir V Sinitsin ${ }^{\mathrm{b}}$ \\ ${ }^{\mathrm{b}}$ South Ural State University (SUSU) \\ Chelyabinsk, Russia. \\ a160403@gmail.com
}

\begin{abstract}
An earlier study described a wireless acceleration sensor used to perform condition monitoring of rotating machinery, via off-line spectral analysis. In this paper, a new technique called Prism signal processing is applied to the same problem to provide sample-by-sample analysis of the machine rotation. The Prism has low design cost and low computational cost, and so is particularly suited to the flexible signal processing requirements for the Internet of Things. This paper provides an overview of the sensor and its experimental rig as well as the Prism-based signal processing scheme used to track the key components for diagnostic monitoring. The validity of the signal processing scheme is demonstrate via a simulation.
\end{abstract}

Keywords- Prism signal processing, diagnostics, accelerometer, wireless acceleration sensor, rotating machinery.

\section{INTRODUCTION}

Modern trends in Industry 4.0 such as smart and flexible manufacturing systems require enhanced reliability and operational predictability of their nodes and components. Fault detection and diagnosis methods are under intense development for instruments in industrial systems. The implementation of these methods improves machine condition monitoring and leads to a reduction in maintenance costs and the number of unplanned shutdowns.

Vibration-based monitoring is a widely used approach for fault detection and diagnosis in rotating machines. The most common methods for fault diagnosis compare, via various signal parameters (for example, RMS value, peak amplitude or kurtosis), the 'healthy machine state' and the 'current machine state'. However, different machine workloads may lead to different vibration levels causing such methods to lose efficiency [1]. More specialized methods have been developed for the fault diagnosis of machines with fluctuation loads, for example, the combination of Time Synchronous Averaging (TSA) with the complex Morlet wavelet [2], the Hilbert transform [3], or the MIR-TSA technique [4]. Unfortunately, these methods have a limitation of machine fluctuation speed.

Methods based on time-frequency resolution are often more efficient, for example, Empirical Mode Decomposition (EMD) which follows Hilbert Transform (HT) [5] or Hilbert spectrum

The work was supported by Act 211 Government of the Russian Federation, contract № 02.A03.21.0011 based on the maximal overlap discrete wavelet packet transform (MODWPT) [6]. However, these methods require a high level of computational resources.

A new signal processing object, the Prism [7], matches these requirements by having trivial design cost (so that new signal processing schemes can be instantiated in real time to match changing signal characteristics) and a low computing cost per sample. As summarised in [7], networks of Prisms can carry out a variety of signal processing tasks such as low pass, bandpass and notch filtering, the 'splitting' of signals into separate frequency components, and the tracking of frequency, phase and amplitude information. Previous applications include Coriolis mass flow metering [8], where Prism signal processing has facilitated a significant increase in the measurement update rate to $48 \mathrm{kHz}$, enabling for the first time direct measurement of diesel engine fuel injection; pressure sensor diagnostics [9] where ultrasonic pulses composed of multiple decaying sinusoids are decomposed to provide diagnostic data; and sensor validation more broadly [10], whereby the presence of an undesired frequency component in a transducer signal is detected, isolated, tracked, and compensated, within one second of the fault occurring.

Strongly associated with Industry 4.0 [11] is the Internet of Things (IoT) [12] which proposes a substantial increase in the deployment of sensors - for collecting, processing and communicating measurement data in real time - in diverse working environments. The implementation of the IoT concept alongside wireless technologies will support the development of sensors with unique functions, which accordingly can improve condition monitoring. An example of a wireless technology implementation is the Wireless Acceleration Sensor (WAS) [13]. WAS contains several MEMS-accelerometers and is mounted on the moving element (for example, the rotating shaft) of a machine so that accelerometer data (and eventually power) may be transferred wirelessly. Thus, WAS is more flexible, and potentially more sensitive than traditional technologies. Moreover, WAS can measure angular as well as linear accelerations of the moving element.

This paper describes the application of Prism signal processing to a WAS application where the WAS is mounted on the moving element of a rotating test rig. The signal 
processing scheme has two stages. The first stage tracks the frequency of the variable speed rotation, despite the presence of multiple harmonics in the signal. The second stage provides a series of bandpass filters and trackers to monitor the frequency and amplitude of each frequency component once the rotor has established a steady rotation. These sinusoidal component parameters may be used to perform diagnostic analysis of the rotor operation.

A key feature of the Prism signal processing scheme is that the bandpass filtering can be (re)designed around the rotor's current operating frequency with minimum design effort and with no specialized design software. This demonstrates a solution to one of the challenges identified in [7] for the IoT low cost, localized redesign of the signal processing scheme in response to changing requirements.

This paper presents the signal processing scheme, and demonstrates via a simulation exercise that the component parameters can be tracked to reasonable accuracy. A companion conference paper provides experimental data for faulty and non-faulty conditions to demonstrate the technique's applicability to the real system. Future work will include the development of diagnostic reasoning based on the amplitude and frequency data obtained via Prism signal processing, as well as a real-time implementation of the scheme.

\section{WIRELESS ACCELEROMETER}

The WAS prototype (Fig. 1), developed at SUSU [13], uses three one-axis MEMS-accelerometers (ADXL001-70 from Analog Devices) as sensing elements. Sampling is performed at $41 \mathrm{kHz}$ using an AD7609 8-channel simultaneous sampling ADC. The sensing system is controlled by the ARM-based power micro-controller STM32L476 (STMicroelectronics), which communicates wirelessly via an RF receiver-transmitter. The experimental sensor uses lithium batteries for power, but in the longer term wireless power transfer is envisaged.

The PCB sensing board has been evaluated using an experimental rig, supplied by SpectraQuest, which is designed to simulate a range of typical machine faults, such as load imbalance and misalignment. The sensing board was mounted on the end of the shaft (figure 2).

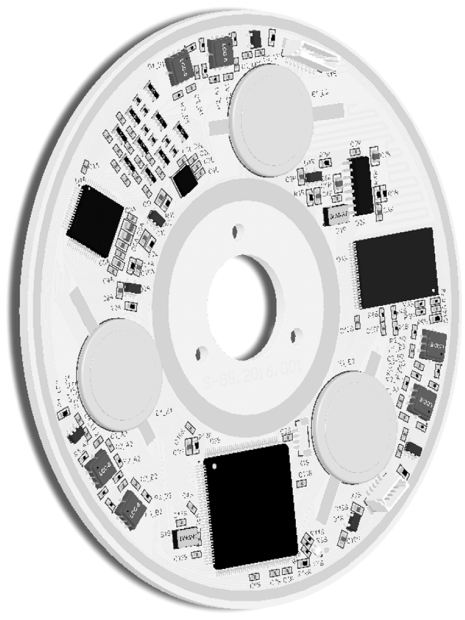

Fig. 1. PCB accelerometer card (from [13])

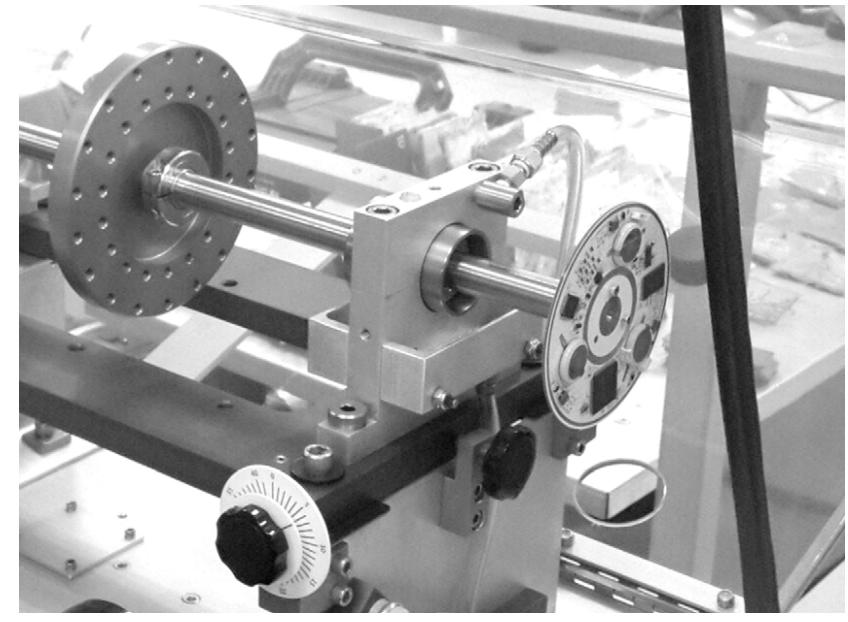

Fig. 2. PCB sensing board mounted on shaft of experimental rig (from [13])

In [13], a summary is provided of the basic signal processing used to extract the angular acceleration of the shaft from the three sensor signals. Fig. 3 shows a power spectrum analysis of the angular acceleration signal for an experiment in which the rotor starts at rest and accelerates to a steady rotation of around $12 \mathrm{~Hz}$. This shows a series of peaks up to the twelfth harmonic of the rotation frequency, with highest amplitudes at the fourth and sixth harmonic. When the rotor is oscillating steadily, the harmonics are retained but the power spectrum noise floor is relatively flat. The distortions below $80 \mathrm{~Hz}$ in Figure 3 is due to the variation in rotational speed as the rotor initiates motion and accelerates to a steady rotation.

The relative amplitudes of the harmonics could potentially form a set of diagnostic parameters to be used for detecting faults. However, such analysis would preferably be implemented in real-time on a sample-by-sample basis, and would be further capable of tracking the operation of the rotor through transitions, including starting and stopping, where fault conditions might have particular characteristics.

In this paper we describe a Prism signal processing scheme to track the variable speed of the rotor, and then to further extract the harmonic characteristics of the angular acceleration signal for further diagnostic analysis. A simulation demonstrates the operation of the scheme. In our companion conference paper, we give examples of experimental results where fault modes have been induced in the rotor system in order to demonstrate the performance of the proposed signal processing scheme on real data.

\section{PRISM SignAL Processing}

The Prism is a signal processing object which takes an input time series and generates one or two output time series. Mathematical details of Prism implementation are discussed in [7-10]. The Prism behaves like a pair of FIR filters operating over a window of input data of duration $2 / m$, where $m$ is the characteristic frequency of the Prism. However, the Prism has two significant advantages over conventional FIR filters. Firstly, the Prism has a recursive calculation, so that the computation per sample is low, and independent of the Prism window length. This is well suited to the restricted 
computational budgets of IoT devices. Secondly, filter design is simple, as the 'coefficients' are just linearly spaced sine and cosine values. Accordingly, any device capable of running a Prism should be capable of designing or redesigning it as signal properties or operational requirements change over time.

Networks of Prisms can be used to perform a range of filtering tasks, including low pass, bandpass and (static) notch filtering. In addition, Prism-based trackers can be used to generate sample-by-sample estimates of frequency, amplitude and/or phase of a sinusoid. These trackers share the Prism characteristics of low computational cost and simple design. For example, Prism-based pre-filtering and tracking of transducer data has been implemented to achieved a significantly increased measurement update rate (up to $48 \mathrm{kHz}$ ) for Coriolis mass flow measurement [8].

Here, we demonstrate the flexibility of Prism signal processing, using the accelerator data from the experimental rotor system. The key goal is as follows: to track the frequency and amplitude of up to eight signal components, allowing for variable rotor speed between say $10 \mathrm{~Hz}$ and $18 \mathrm{~Hz}$. The amplitude data may be used to perform diagnostics to detect a variety of fault conditions. However, the focus here is on the basic Prism signal processing scheme to generate the amplitude data. It is assumed that the test equipment will operate over a series of 'runs' as exemplified by Fig. 3. The sampling (and measurement) update rate to be managed is $41 \mathrm{kHz}$.

A simple means of achieving this goal is to use suitably designed bandpass filtering and tracking to isolate each signal component. However, the key criterion for determining the bandpass filter design is the steady rotation speed, which is arbitrary within given limits, and unknown. Accordingly, the signal processing developed here consists of two stages:

- A first stage which tracks the rotational frequency (only) during the startup period. This stage must be

able to operate over the full range of rotor frequencies.

- A second stage to track all component amplitudes and frequencies once the steady rotor operation has commenced. The key here is that the signal processing is re-designed each run based around the steady rotational speed as determined by the first stage.

Here the signal processing scheme is outlined, and simulation results demonstrate its ability to track multiple components. In the companion paper, the system is applied to experimental data for a variety of rotational speeds and fault conditions.

\section{Simulation OF ACCELEROMETER DATA}

Table I lists the typical structure of experimental angular acceleration signals, as illustrated in Figure 3. Here $f$ is the instantaneous operating frequency. The $4^{\text {th }}$ harmonic has the largest amplitude, followed by the $6^{\text {th }}$ harmonic.

TABLE I. Simulated ANGUlar ACCELERATION Signal COMPONENTS

\begin{tabular}{|c|c|c|}
\hline \multirow{2}{*}{ Index } & \multicolumn{2}{|c|}{ Sinusoidal properties } \\
\cline { 2 - 3 } & Frequency $(\mathrm{Hz})$ & Amplitude $\left(\mathrm{rad} / \mathbf{s}^{\mathbf{2}}\right)$ \\
\hline 1 & $f$ & 2 \\
\hline 2 & $2 f$ & 3 \\
\hline 3 & $3 f$ & 4 \\
\hline 4 & $4 f$ & 100 \\
\hline 5 & $5 f$ & 2 \\
\hline 6 & $6 f$ & 40 \\
\hline 7 & $8 f$ & 2 \\
\hline 8 & $12 f$ & 2 \\
\hline
\end{tabular}

ypical Experimental Data
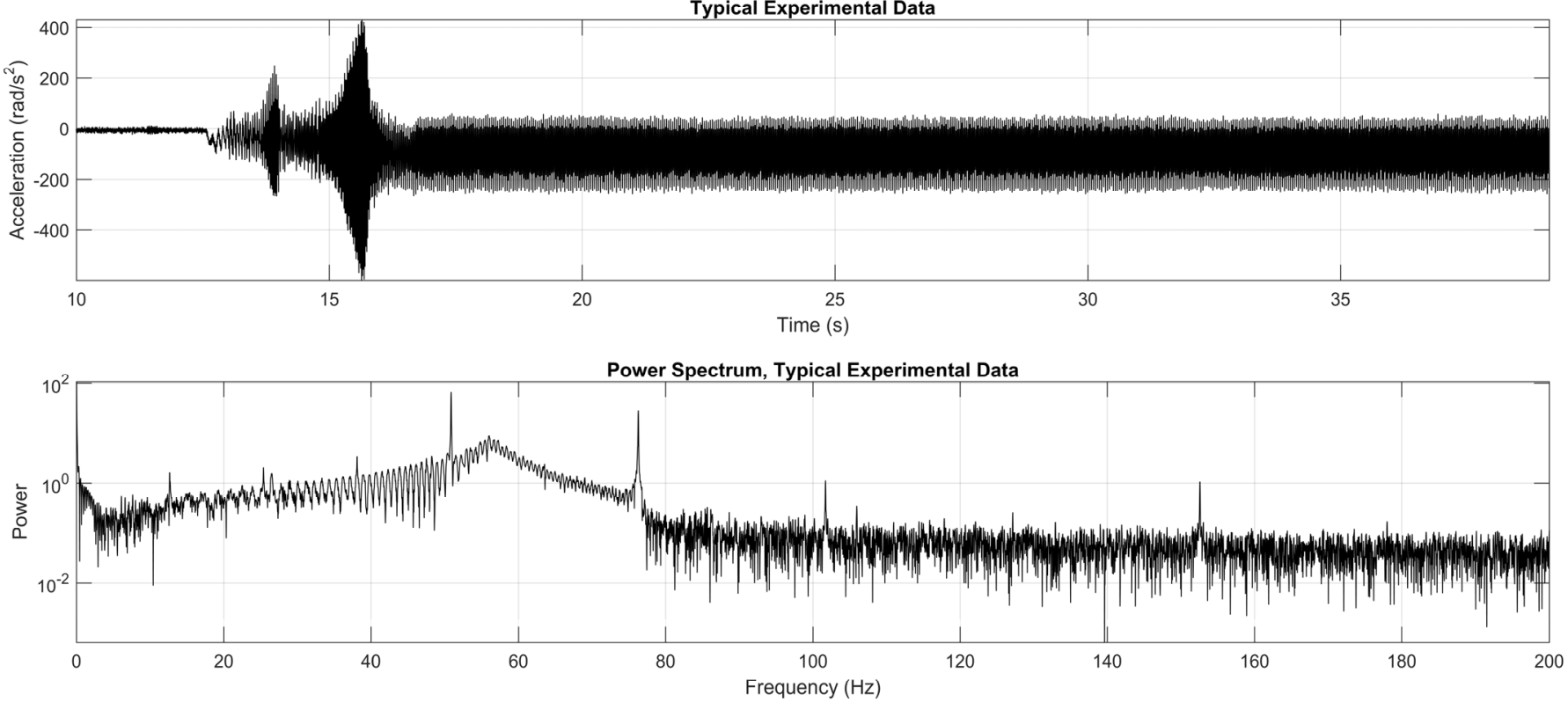

Fig. 3. Typical angular acceleration from rotor experiment 

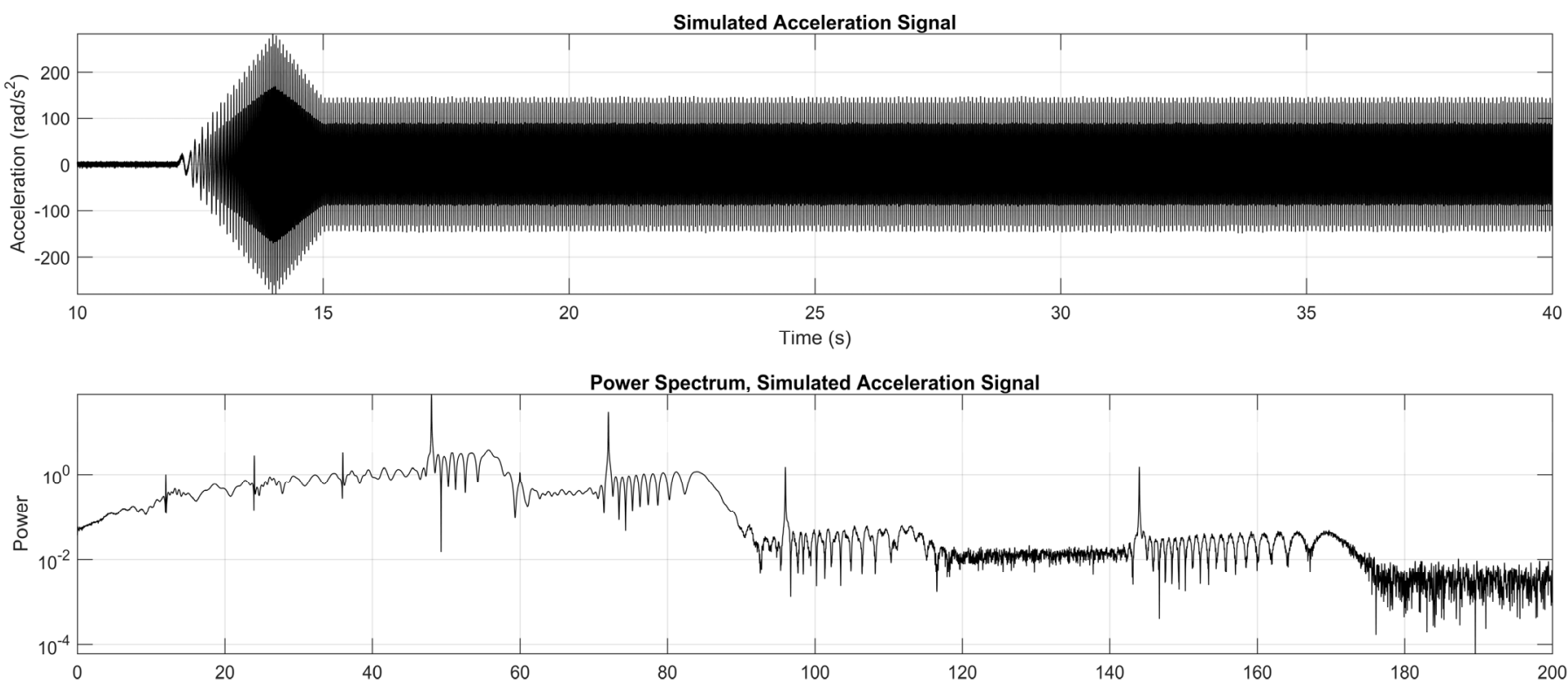

Fig. 4. Simulated angular acceleration data

Note that this signal structure is typical for steady operation, but the true signal structure is unknown during startup, when the frequencies and amplitudes move from zero to their steady operating values. For simplicity, it is assumed for simulation purposes that the amplitude and frequency ratios remain the same. A white noise component with standard deviation 2 $\mathrm{rad} / \mathrm{s}^{2}$ is included in the simulated signal.

For example, Figure 4 shows a simulated startup signal, which has linear changes in amplitude and frequency occuring in two parts. Firstly there is an initial linear rise from zero (from $t=12 \mathrm{~s}$ to $\mathrm{t}=14 \mathrm{~s}$ ), which overshoots the steady state frequency and amplitude. This is followed by a linear reduction in frequency and amplitude (from $t=14 \mathrm{~s}$ to $t=15 \mathrm{~s}$ ) to reach the steady state values $(\mathrm{t}>15 \mathrm{~s})$. This two part initalisation is a simplified version of actual experimental results, as shown in the companion paper.
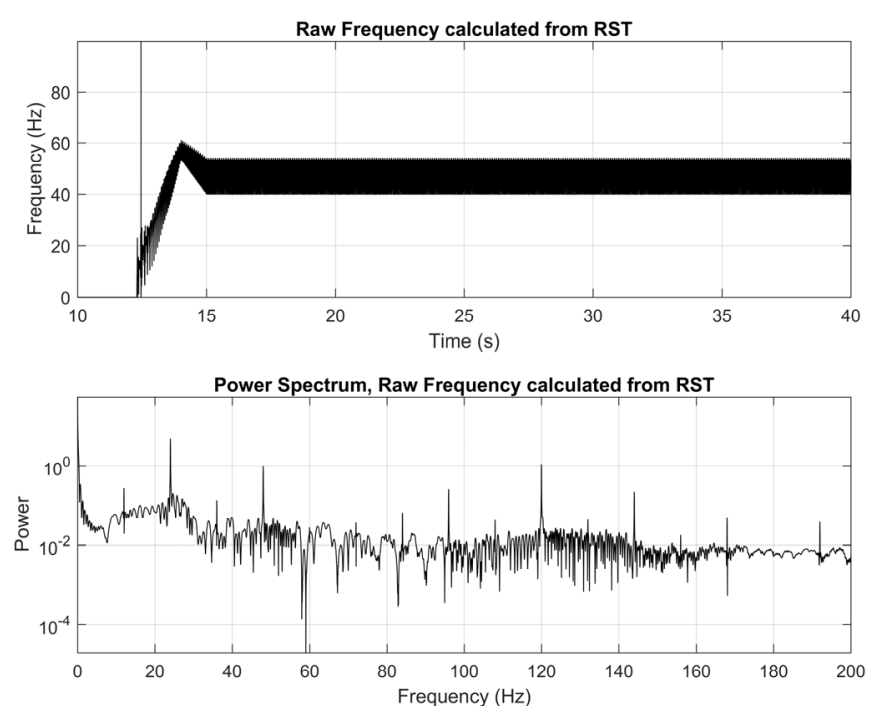

Fig. 5. RST tracking of $4^{\text {th }}$ harmonic rotor frequency from simulation data

\section{Stage 1: FREQUENCY TRACKING}

The first stage of the signal processing is required to track the frequency of the rotor through the intialisation period, in order to establish the steady state frequency. Reference [9] outlines the operation of the Prism-based Recursive Signal Tracker (RST), which tracks the frequency, amplitude and phase of an input sinusoid on a sample-bysample basis. However, its working assumption is that the input signal consists of a single sinusoidal component plus white noise. When additional signal components are present, appropriate further steps are required to ensure successful tracking. Figure 5 shows the raw frequency results obtained from simply applying the RST to the signal data. The results track the trend of the simulated data - a linear rise past the steady frequency, a drop back, and then, for $\mathrm{t}>15 \mathrm{~s}$, a 'steady' frequency value, corresponding to that of the largest harmonic, at $48 \mathrm{~Hz}$. However, the frequency measurement is clearly very
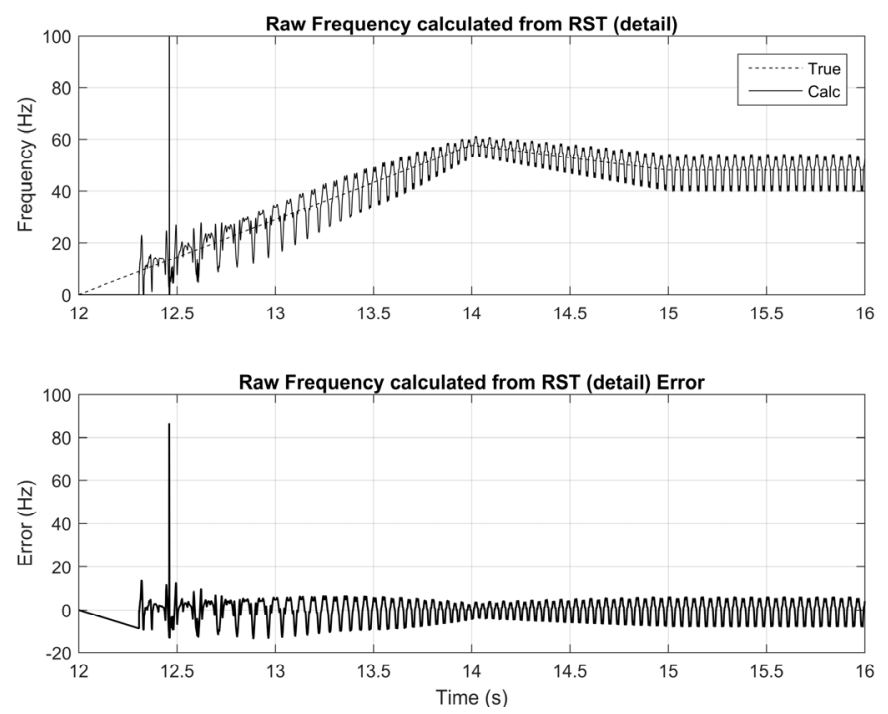

Fig. 6. RST tracking of $4^{\text {th }}$ harmonic rotor frequency (detail) 

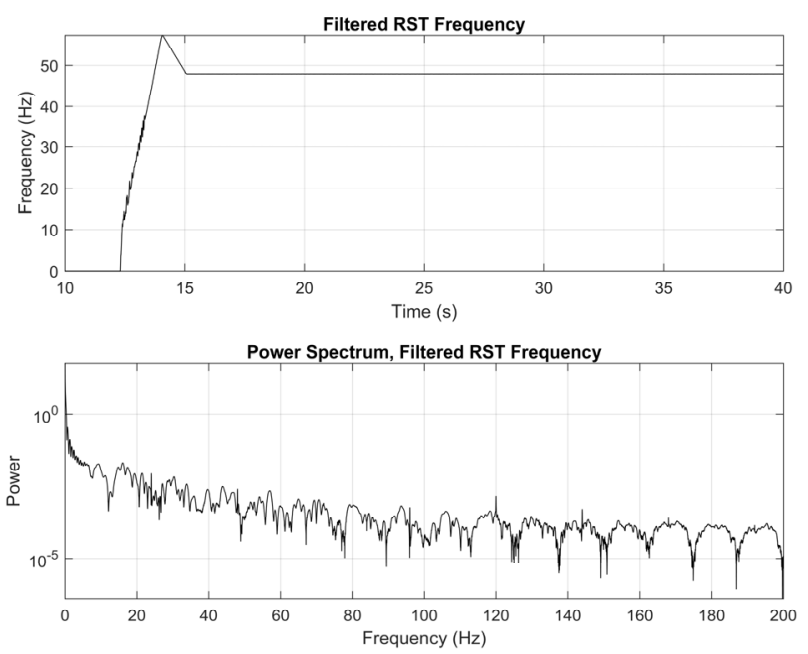

Fig. 7. Filtered RST rotor $4^{\text {th }}$ harmonic frequency

noisy, due to interference from the other components. The frequency power spectrum indicates the noise structure, with peaks at multiples of the steady rotation frequency, $12 \mathrm{~Hz}$. Fig. 6 shows the true frequency over the initiation period $(12 \mathrm{~s}-$ $16 \mathrm{~s}$ ), along with the measured frequency and its error. While the RST measurement successfully follows the trend of the true frequency, the error signal shows large oscillations, corresponding to the peaks in the power spectrum of Fig. 5. This structure suggests a simple form of noise-reducing filtering - using an FIR moving average filter with a window length corresponding to the period of the first harmonic. Note that it is straightforward to create a variable length moving average filter which adapts its length to match the period of the (average) observed frequency or its sub-multiple. Figs. 7 and 8 show the results of applying the moving average filtering to the raw frequency data. Fig. 7 shows the complete time series and power spectrum, while Fig. 8 shows the filtered measurement and its error over the initiation period. The filtering results in a significant reduction in frequency error. This signal processing scheme serves to track the rotor operaton during the startup phase, including the identification of the steady operating frequency, which is used to design the

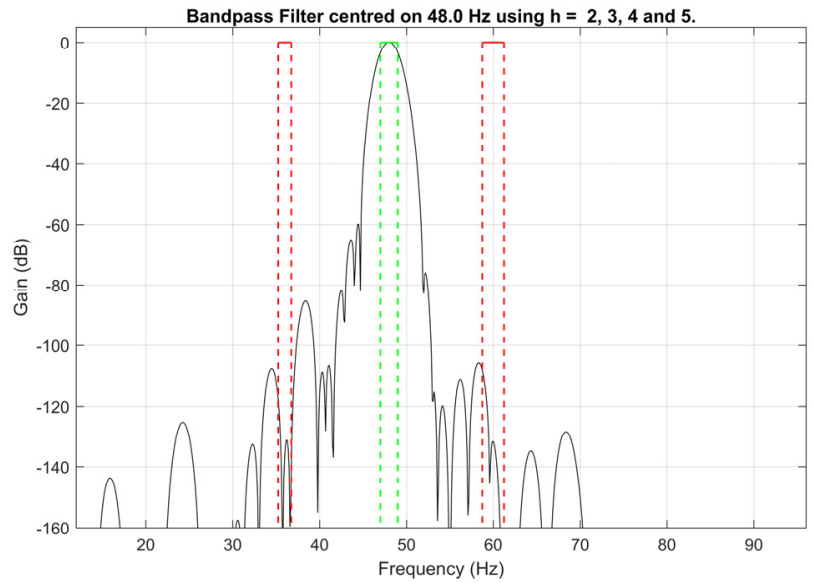

Fig. 9. Prism-based bandpass filter design centred on $48 \mathrm{~Hz}$
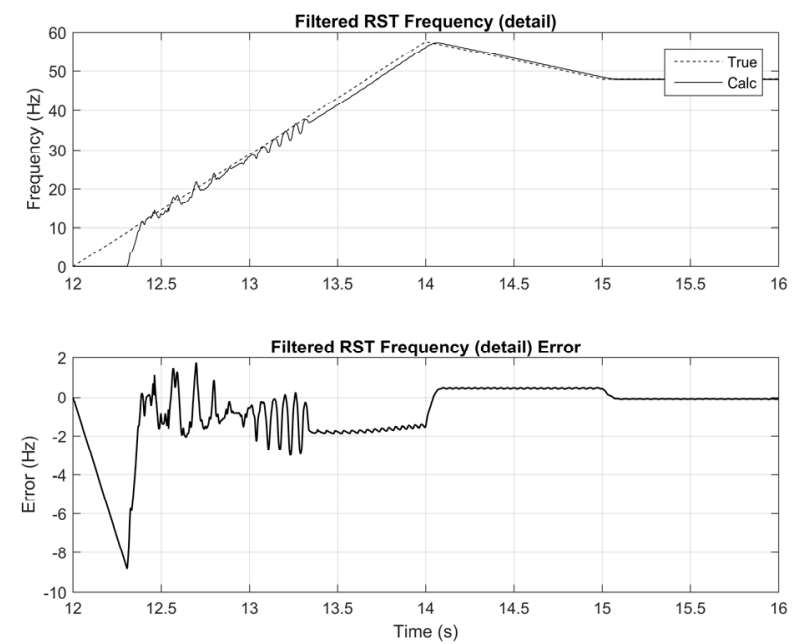

Fig. 8. Filtered RST rotor $4^{\text {th }}$ harmonic frequency (detail)

second stage signal processing scheme.

\section{StAge 2: TRACKING ALL COMPONENTS}

In the second stage of rotor operation, the amplitudes and frequencies of all the signal components are tracked. The simplest way of achieving this is to use narrow bandpass filters around the frequency of each component, followed by an RST tracking block to extract amplitude and frequency information. As discussed in [7], Prism-based bandpass filtering has two key advantages. Firstly, the design cost is low, so that it is perfectly feasible to design bandpass filters in real time based around the current operating frequency of the rotor. Secondly, the computational cost of applying the filter is also low, which again makes it well suited to real-time and IoT applications. Figure 9 shows the frequency response of a bandpass filter consisting of a chain of four pairs of Prisms (as described in [7]) with harmonic numbers $h$ of 2, 3, 4, and 5 respectively. Centred on $48 \mathrm{~Hz}$, the $4^{\text {th }}$ harmonic frequency in this simulation, it provides a pass band of around $\pm 1 \mathrm{~Hz}$, while adjacent higher and lower harmonic frequencies are significantly attenuated.

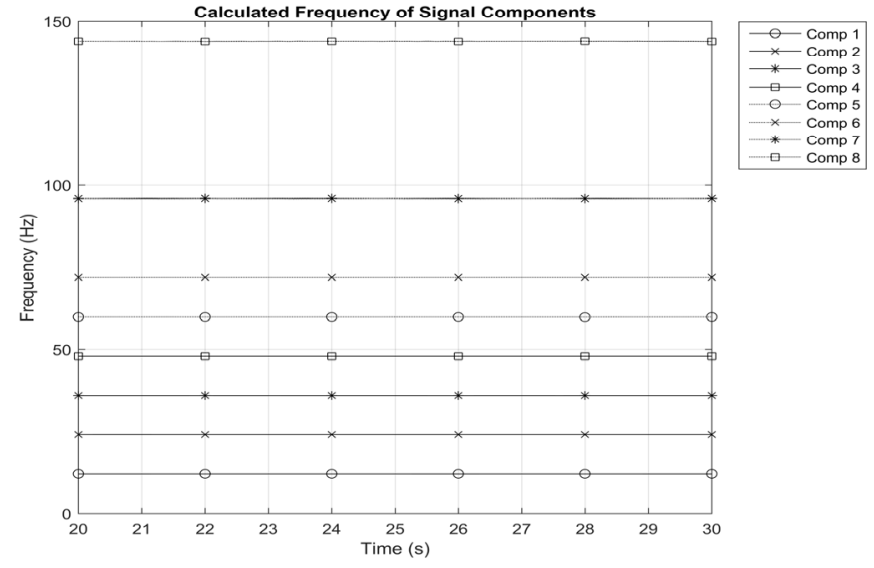

Fig. 10. Frequency tracking - all components. 


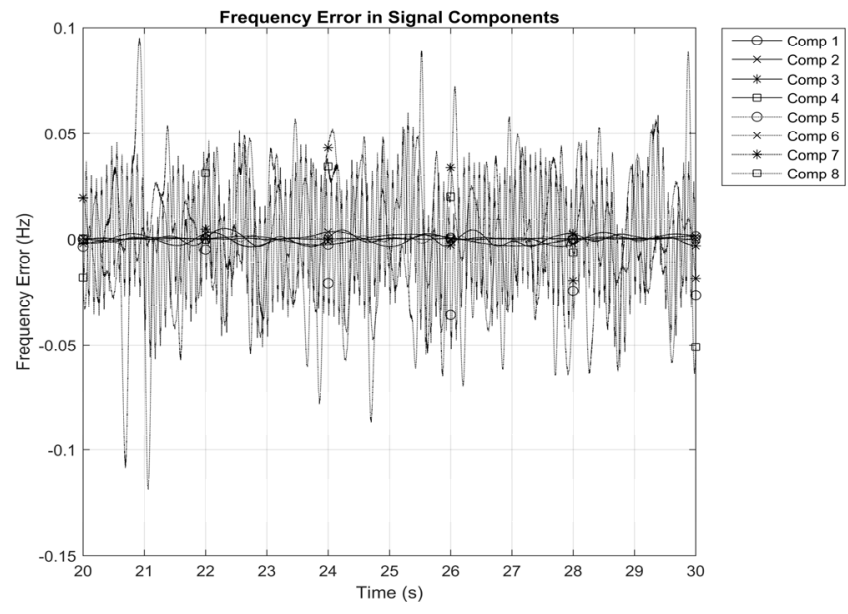

Fig. 11. Frequency tracking error - all components.

Bandpass filtering followed by RST tracking is applied to each frequency component, where the central frequency of the bandpass filter along with the characteristic frequency $m$ of the RST are selected appropriately. Figs. 10 and 11 show the frequency and amplitude values tracked for each of the signal components for this simulation during a period of steady operation, while Figs. 12 and 13 show the corresponding errors. Note that all measurement values are generated once per sample at $41 \mathrm{kHz}$. The frequency errors fall mostly within $\pm 0.1 \mathrm{~Hz}$, while the amplitude errors all mostly within \pm 0.1 $\mathrm{rad} / \mathrm{s}^{2}$. This tracking performance is considered satisfactory for the purposes of the experimental work. Similar results are obtained for different steady rotational frequencies in the range $8 \mathrm{~Hz}$ to $20 \mathrm{~Hz}$, matching the operating range of the equipment. The companion paper reports on the results obtained when applying this signal processing scheme to experimental data, in particular for the purposes of fault detection.

\section{DISCUSSION}

The IoT requires fast and flexible signal processing techniques. A key to flexibility is rapid and low-cost filter
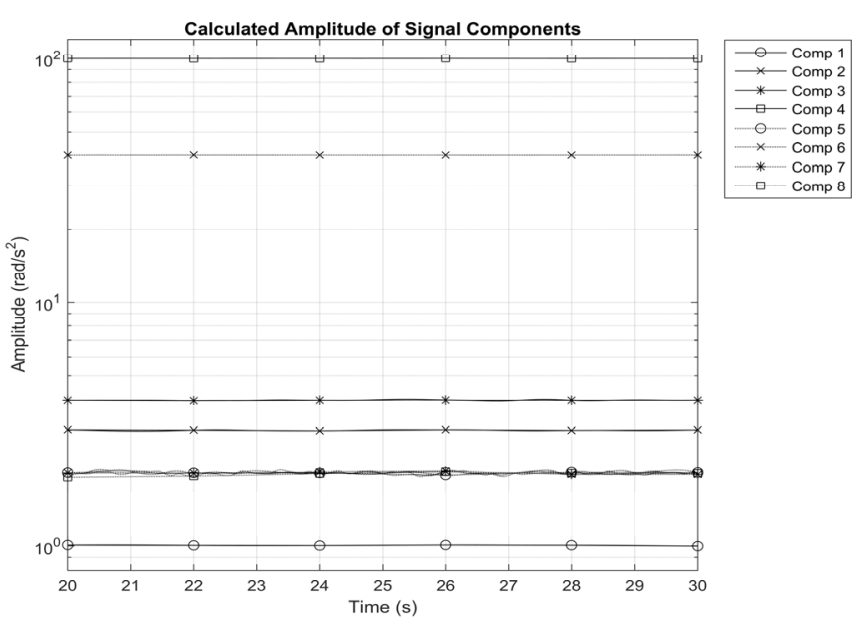

Fig. 12. Amplitude tracking - all components.

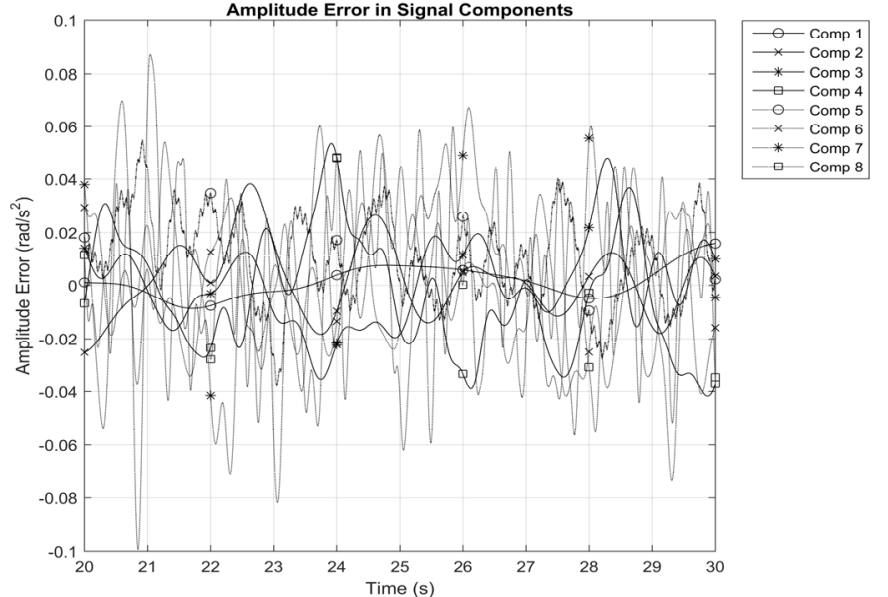

Fig. 13. Amplitude tracking errors - all components.

design that can be carried out in real time in order to adapt to current signal properties. This paper has provided a simple worked example of how Prism signal processing can provide the required flexibility and low-cost real-time design.

\section{REFERENCES}

[1] C.J. Stander, P.S.Heyns, W.Schoombie, Using vibration monitoring for local fault detection on gears operating under fluctuating load conditions, Mech. Syst. Signal Process. 16(6) (2002) 1005-1024.

[2] M.A. Jafarizadeh, R. Hassannejad, M.M. Ettefagh, S. Chitsaz, Asynchronous input gear damage diagnosis using time averaging and wavelet filtering, Mech. Syst. Signal Process., Vol. 22, 2008, pp 172201, DOI: 10.1016/j.ymssp.2007.06.006

[3] M. Feldman, Hilbert transform in vibration analysis, Mech. Syst. Signal Process., 25(3), 2011, pp 735-802, DOI: 10.1016/j.ymssp.2010.07.018

[4] N. Ahamed, Y. Pandya, A. Parey, Spur gear tooth root crack detection using time synchronous averaging under fluctuating speed, Measurement, Vol. 52, 2014, pp 1-11.

[5] M. Amarnath, I.R. Praveen Krishna, Local fault detection in helical gears via vibration and acoustic signals using EMD based statistical parameter analysis, Measurement, Vol. 58, 2014, pp 154-164.

[6] Yu Yang, Yigang He, Junsheng Cheng, Dejie Yu, A gear fault diagnosis using Hilbert spectrum based on MODWPT and a comparison with EMD approach, Measurement, Vol. 42, 2009, pp 542-551, DOI: 10.1016/j.measurement.2008.09.011

[7] M.P. Henry, F. Leach, M. Davy, O. Bushuev, M.S. Tombs, F. B. Zhou, and S. Karout, "The Prism: Efficient Signal Processing for the Internet of Things", IEEE Industrial Electronics Magazine, pp 2-10, December 2017. DOI: 10.1109/MIE.2017.2760108.

[8] F. Leach, S. Karout, F.B. Zhou, M.S. Tombs, M. Davy, and M.P. Henry, "Fast Coriolis mass flow metering for monitoring diesel fuel injection", Flow Measurement and Instrumentation, 58 (2017), pp 1-5.

[9] Henry, MP, Bushuev, O, Ibryaeva, O. "Prism Signal Processing for Sensor Condition Monitoring", 26th IEEE International Symposium on Industrial Electronics, Edinburgh, UK, 2017.

[10] Henry, MP. "An Introduction to Prism Signal Processing applied to Sensor Validation", Measurement Techniques, pp 1233 - 1237, Mar 2018. DOI: $10.1007 / \mathrm{s} 11018-018-1345-1$

[11] B. Vogel-Heuser, D. Hess, "Guest Editorial - Industry 4.0-Prerequisites and Visions", IEEE Transactions on Automation Science and Engineering, Vol 14, No. 2, April 2016.

[12] M. Wollschlaeger, T. Sauter, and J. Jasperneite, "The Future of Industrial Communication", IEEE Industrial Electronics Magazine, pp. 17-27, March 2017. DOI : 10.1109/MIE.2017.2649104

[13] V. Sinitsin and A. L. Shestakov, "Wireless acceleration sensor of moving elements for condition monitoring of mechanisms", Meas. Sci. Technol. 28 (2017) 094002. https://doi.org/10.1088/1361-6501/aa7ab6 